\title{
CORRECTION
}

\section{Correction: Mediators of racial and ethnic disparity in mother's own milk feeding in very low birth weight infants}

\author{
Aloka L. Patel ${ }^{1}$, Michael E. Schoeny ${ }^{2}$, Rebecca Hoban ${ }^{3}$, Tricia J. Johnson ${ }^{4}$, Harold Bigger ${ }^{1}$, Janet L. Engstrom ${ }^{2}$, Erin Fleurant ${ }^{5}$, \\ Brittany Riley ${ }^{6}$ and Paula P. Meier ${ }^{1}$
}

Pediatric Research (2019) 86:786; https://doi.org/10.1038/s41390-019-0576-4

Correction to: Pediatric Research (2019) 85, 662-670 https://doi.org/ 10.1038/s41390-019-0290-2, published online 18 January 2019.

In the original version of this article, the results for the two MOM Pumping Factors in Table 2 were inadvertently reversed. The values for 'Mean daily pumping frequency (first 14 days)' were accidentally placed in the row 'Coming to volume by day 14', and vice versa. The corrected version of Table 2 is displayed here. This has not been corrected in the PDF or HTML versions of the article.
Table 2. Composite logistic regression of factors and MOM feeding at NICU discharge $(N=415)$

\begin{tabular}{|c|c|c|c|c|c|c|}
\hline & \multicolumn{3}{|c|}{ Step $1^{a}$} & \multicolumn{3}{|c|}{ Step $2^{\mathrm{a}}$} \\
\hline & OR & \multicolumn{2}{|c|}{$95 \% \mathrm{Cl}$} & OR & \multicolumn{2}{|c|}{$95 \% \mathrm{Cl}$} \\
\hline \multicolumn{7}{|l|}{ Demographic factors } \\
\hline Maternal age $\mathrm{e}^{* *}$ & - & - & - & 1.09 & 1.03 & 1.14 \\
\hline $\begin{array}{l}\text { Low socioeconomic status } \\
\text { (WIC eligible)* }\end{array}$ & - & - & - & 0.38 & 0.16 & 0.91 \\
\hline Married & - & - & - & 1.03 & 0.50 & 2.08 \\
\hline \multicolumn{7}{|l|}{ Race/ethnicity (Black as reference) } \\
\hline White/Asian & - & - & - & 0.91 & 0.37 & 2.25 \\
\hline Hispanic ${ }^{* *}$ & - & - & - & 3.01 & 1.33 & 6.81 \\
\hline \multicolumn{7}{|l|}{$\begin{array}{l}\text { Highest education (<High School as } \\
\text { reference) }\end{array}$} \\
\hline High School graduate & - & - & - & 0.95 & 0.64 & 1.40 \\
\hline Trade school or $<\mathrm{BA} / \mathrm{BS}^{*}$ & - & - & - & 1.49 & 1.07 & 2.08 \\
\hline $\mathrm{BA} / \mathrm{BS}$ or higher & - & - & - & 1.19 & 0.89 & 1.59 \\
\hline Number of children in household ${ }^{\mathrm{b}}$ & - & - & - & 0.54 & 0.27 & 1.11 \\
\hline \multicolumn{7}{|l|}{ Social factors } \\
\hline Initial goal of any MOM & 1.97 & 0.82 & 4.75 & 2.31 & 0.69 & 7.70 \\
\hline $\begin{array}{l}\text { Breastfeeding support from mother's } \\
\text { mother* }\end{array}$ & 0.56 & 0.34 & 0.90 & 0.50 & 0.28 & 0.91 \\
\hline Maternal smoking & 0.85 & 0.50 & 1.46 & & & \\
\hline \multicolumn{7}{|l|}{ Neighborhood structural factors } \\
\hline Access to a car & 1.19 & 0.68 & 2.09 & & & \\
\hline \multicolumn{7}{|l|}{ Maternal health and infant factors } \\
\hline Multiple gestation & 0.86 & 0.43 & 1.71 & & & \\
\hline Pre-eclampsia/eclampsia & 0.62 & 0.36 & 1.07 & 0.65 & 0.32 & 1.33 \\
\hline Infant male sex & 1.08 & 0.69 & 1.71 & & & \\
\hline Infant gestational age & 1.10 & 1.00 & 1.21 & 1.05 & 0.93 & 1.20 \\
\hline 5-Min APGAR score ${ }^{b}$ & 1.02 & 0.57 & 1.81 & & & \\
\hline \multicolumn{7}{|l|}{ MOM pumping factors } \\
\hline $\begin{array}{l}\text { Mean daily pumping frequency (first } \\
14 \text { days)** }\end{array}$ & 1.90 & 1.46 & 2.47 & 1.70 & 1.26 & 2.29 \\
\hline Coming to volume by day $14^{\dagger}$ & 9.03 & 4.26 & 19.13 & 7.46 & 3.26 & 17.07 \\
\hline \multicolumn{7}{|c|}{$\begin{array}{l}B A / B S \text { Bachelor of Arts/Science, MOM mother's own milk, NICU neonatal } \\
\text { intensive care unit, WIC Special Supplemental Nutrition Program for } \\
\text { Women, Infants and Children } \\
\text { For Step } 2:{ }^{*} p<0.05 ;{ }^{* *} p<0.01 ;{ }^{+} p<0.001 \\
{ }^{\text {a }} \text { Step } 1 \text { tested individual factors (social, neighborhood structural, maternal } \\
\text { health and infant, and MOM pumping) controlling for demographics. Factors } \\
\text { in bold in Step } 1 \text { were significant at } p<0.15 \text { and were included in Step } 2 \\
{ }^{b} \text { Log-transformed values used for analysis }\end{array}$} \\
\hline
\end{tabular}

${ }^{1}$ Department of Pediatrics, Rush University Children's Hospital, Chicago, LL, USA; ${ }^{2}$ College of Nursing, Rush University Medical Center, Chicago, IL, USA; ${ }^{3}$ Department of Paediatrics, Division of Neonatology, Hospital for Sick Children, Toronto, ON, Canada; ${ }^{4}$ Department of Health Systems Management, Rush University, Chicago, IL, USA; ${ }^{5}$ Department of Obstetrics and Gynecology, University of Connecticut Health, Farmington, CT, USA and ${ }^{6}$ Department of Neonatology, Nationwide Children's Hospital, Columbus, OH, USA Correspondence: Aloka L. Patel (Aloka_Patel@Rush.edu)

Published online: 18 September 2019 